\title{
ON THE INFERENCE ABOUT THE SPECTRAL DISTRIBUTION OF HIGH-DIMENSIONAL COVARIANCE MATRIX BASED ON HIGH-FREQUENCY NOISY OBSERVATIONS
}

\author{
By Ningning XiA ${ }^{1}$ AND XinghuA ZHENG ${ }^{2}$ \\ Shanghai University of Finance and Economics and Hong Kong University of \\ Science and Technology
}

\begin{abstract}
In practice, observations are often contaminated by noise, making the resulting sample covariance matrix a signal-plus-noise sample covariance matrix. Aiming to make inferences about the spectral distribution of the population covariance matrix under such a situation, we establish an asymptotic relationship that describes how the limiting spectral distribution of (signal) sample covariance matrices depends on that of signal-plus-noisetype sample covariance matrices. As an application, we consider inferences about the spectral distribution of integrated covolatility (ICV) matrices of high-dimensional diffusion processes based on high-frequency data with microstructure noise. The (slightly modified) pre-averaging estimator is a signal-plus-noise sample covariance matrix, and the aforementioned result, together with a (generalized) connection between the spectral distribution of signal sample covariance matrices and that of the population covariance matrix, enables us to propose a two-step procedure to consistently estimate the spectral distribution of ICV for a class of diffusion processes. An alternative approach is further proposed, which possesses several desirable properties: it is more robust, it eliminates the effects of microstructure noise, and the asymptotic relationship that enables consistent estimation of the spectral distribution of ICV is the standard Marčenko-Pastur equation. The performance of the two approaches is examined via simulation studies under both synchronous and asynchronous observation settings.
\end{abstract}

\section{Introduction.}

1.1. Motivation. Covariance structure is of fundamental importance in multivariate analysis and applications. While in the classical low-dimensional setting, a usually unknown covariance structure can be estimated by the sample covariance matrix, in the high-dimensional setting, it is now well understood that the

Received August 2015; revised January 2017.

${ }^{1}$ Supported in part by GRF 606811 and 16305315 of the HKSAR, NSFC 11501348, Shanghai Pujiang Program 15PJ1402300, IRTSHUFE and the state key program in the major research plan of NSFC 91546202.

${ }^{2}$ Supported in part by DAG (HKUST) and GRF 606811 and 16305315 of the HKSAR. MSC2010 subject classifications. Primary 62H12; secondary 62G99, 60F15.

Key words and phrases. High-dimension, high-frequency, integrated covariance matrices, Marčenko-Pastur equation, microstructure noise. 
sample covariance matrix is not a consistent estimator. Furthermore, in many applications the observations are contaminated. Below, we explain one such setting that motivates this work. Similar situations arise in many other settings, especially in signal processing [see, e.g., Dozier and Silverstein (2007a), El Karoui (2010b), and Hachem et al. (2012)].

Our motivating question arises in the context of estimating the so-called integrated covariance matrix of the high-dimensional diffusion process and has applications to the study of stock price processes. More specifically, suppose that we have $p$ stocks whose (latent) log price processes are denoted by $\left(X_{t}^{j}\right)$ for $j=1, \ldots, p$. Let $\mathbf{X}_{t}=\left(X_{t}^{1}, \ldots, X_{t}^{p}\right)^{T}$, where $T$ denotes the transpose. A widely used model for $\left(\mathbf{X}_{t}\right)$ is

$$
d \mathbf{X}_{t}=\boldsymbol{\mu}_{t} d t+\boldsymbol{\Theta}_{t} d \mathbf{W}_{t}, \quad t \in[0,1],
$$

where $\left(\boldsymbol{\mu}_{t}\right)=\left(\mu_{t}^{1}, \ldots, \mu_{t}^{p}\right)^{T}$ is a $p$-dimensional drift process, $\left(\boldsymbol{\Theta}_{t}\right)$ is a $p \times p$ matrix for any $t$ called the covolatility process, and $\left(\mathbf{W}_{t}\right)$ is a $p$-dimensional standard Brownian motion. Both $\left(\boldsymbol{\mu}_{t}\right)$ and $\left(\boldsymbol{\Theta}_{t}\right)$ can be stochastic and depend on the Brownian motion $\left(\mathbf{W}_{t}\right)$. The interval $[0,1]$ is the time period of interest, say, one trading day (= six and a half hours). The integrated covariance (ICV) matrix refers to

$$
\mathrm{ICV}:=\int_{0}^{1} \boldsymbol{\Theta}_{t} \boldsymbol{\Theta}_{t}^{T} d t .
$$

The ICV matrix, in particular, its spectrum (i.e., its set of eigenvalues), plays an important role in financial applications such as factor analysis and risk management.

A classical estimator of the ICV matrix is the so-called realized covariance (RCV) matrix, which relies on the assumption that $\left(\mathbf{X}_{t}\right)$ could be observed at high frequency. More specifically, suppose that $\left(\mathbf{X}_{t}\right)$ could be observed at time points $t_{i}=i / n$ for $i=0,1, \ldots, n$. Then, the RCV matrix is defined as

$$
\mathrm{RCV}=\sum_{i=1}^{n} \Delta \mathbf{X}_{i}\left(\Delta \mathbf{X}_{i}\right)^{T}
$$

where

$$
\Delta \mathbf{X}_{i}=\left(\begin{array}{c}
\Delta X_{i}^{1} \\
\vdots \\
\Delta X_{i}^{p}
\end{array}\right):=\left(\begin{array}{c}
X_{t_{i}}^{1}-X_{t_{i-1}}^{1} \\
\vdots \\
X_{t_{i}}^{p}-X_{t_{i-1}}^{p}
\end{array}\right)
$$

stands for the vector of $\log$ returns over the period $[(i-1) / n, i / n]$. The consistency and central limit theorems for the RCV matrix under such a setting and when the dimension $p$ is fixed are well known; see, for example, Andersen and Bollerslev (1998), Andersen et al. (2001), Barndorff-Nielsen and Shephard (2002), Jacod and Protter (1998), Mykland and Zhang (2006), among others. 
To obtain a better understanding of the above setting, it is instructive to connect it with the usual multivariate analysis setting. In the simplest case, when $\boldsymbol{\mu}_{t} \equiv 0$ and $\boldsymbol{\Theta}_{t} \equiv \boldsymbol{\Theta}$, we have (1) ICV $=\boldsymbol{\Theta} \boldsymbol{\Theta}^{T}$; (2) the returns $\Delta \mathbf{X}_{i} \stackrel{\mathcal{D}}{=} \mathbf{Z}_{i} / \sqrt{n}$, where $\mathbf{Z}_{i} \sim_{\text {i.i.d. }} N(0, \mathrm{ICV})$, and $\mathrm{RCV} \stackrel{\mathcal{D}}{=} \frac{1}{n} \sum_{i=1}^{n} \mathbf{Z}_{i} \mathbf{Z}_{i}^{T}$. In other words, this simplest setting is equivalent to an i.i.d. observation setting in multivariate analysis, where the sample covariance matrix is used to estimate the population covariance matrix. In general, this situation is more complicated because both $\left(\boldsymbol{\mu}_{t}\right)$ and $\left(\boldsymbol{\Theta}_{t}\right)$ can be stochastic and dependent on the underlying Brownian motion.

The so-called market microstructure noise presents another challenge. In practice, the observed prices are always contaminated versions of the latent prices, the error being referred to as market microstructure noise. Such noise is induced by various frictions in the trading process such as the bid-ask spread and the discreteness of price. Despite its small size, market microstructure noise accumulates at high frequency and badly affects inferences about the latent price processes. Liu, Patton and Sheppard (2015) compare various volatility estimators and point out that microstructure noise is not negligible when the sampling frequency is higher than one observation per five minutes.

The following additive model has been widely adopted in recent studies on volatility estimation:

$$
\mathbf{Y}_{t_{i}}=\mathbf{X}_{t_{i}}+\boldsymbol{\varepsilon}_{i}, \quad i=1, \ldots, n,
$$

where $\mathbf{Y}_{t_{i}}=\left(Y_{t}^{1}, \ldots, Y_{t}^{p}\right)^{T}$ denotes the observations and $\boldsymbol{\varepsilon}_{i}=\left(\varepsilon_{i}^{1}, \ldots, \varepsilon_{i}^{p}\right)^{T}$ denotes the noise, which is i.i.d. independent of $\left(\mathbf{X}_{t}\right)$ with $E\left(\varepsilon_{i}\right)=0$ and certain covariance matrix $\boldsymbol{\Sigma}_{e}$. Observe that under (1.2), the observed log-returns $\Delta \mathbf{Y}_{t_{i}}:=\mathbf{Y}_{t_{i}}-\mathbf{Y}_{t_{i-1}}$ relate to the true log-returns $\Delta \mathbf{X}_{t_{i}}$ by the following equation:

$$
\Delta \mathbf{Y}_{t_{i}}=\Delta \mathbf{X}_{t_{i}}+\Delta \boldsymbol{\varepsilon}_{i}, \quad i=1, \ldots, n,
$$

where, as usual, $\Delta \boldsymbol{\varepsilon}_{i}:=\boldsymbol{\varepsilon}_{i}-\boldsymbol{\varepsilon}_{i-1}$. We are therefore in a noisy observation setting in which the observations are contaminated by additive noise. Such a setting forms the basis of the current work.

One striking feature in (1.3) that differs from most noisy observation settings is that as the observation frequency $n$ goes to infinity, the signal, namely, the true log-return $\Delta \mathbf{X}_{t_{i}}$ becomes diminishingly small, while the noise $\Delta \boldsymbol{\varepsilon}_{i}$ remains being of the same order of magnitude. Therefore, the signal-to-noise ratio goes to 0 . A direct consequence is that even when the dimension is $p=1$, the optimal rate for estimating ICV is only $n^{1 / 4}$ instead of the usual $\sqrt{n}$; see Gloter and Jacod (2001). In other words, due to the dominance of noise over signal, the "effective sample size" is only $O(\sqrt{n})$ rather than $n$. This can be clearly seen from the preaveraging method that we will explain in Section 2.1.

While the problem above constitutes our main motivation for considering a signal-plus-noise observation setting, our results are not restricted to this particular application. Our first main result, Theorem 2.1, applies to a general setting where the signal and noise are of the same order of magnitude. 
1.2. Summary of main results. Our main goal is to make inferences about the spectral distribution of the underlying population covariance matrix, in the setting above, the ICV matrix, based on the noisy observations $\left(\mathbf{Y}_{t_{i}}\right)$ as in (1.2). We provide two approaches, which we summarize as follows.

Approach I requires two steps. We shall introduce an intermediate matrix, $\mathcal{A}_{m}$ as defined in (2.9) below. Think of the ICV matrix as the underlying population covariance matrix. Because our observations are contaminated, the resulting sample covariance matrix is a signal-plus-noise sample covariance matrix. The intermediate matrix $\mathcal{A}_{m}$ is a sample covariance matrix based on only signals. The two steps are then:

- Step 1: derive the Stieltjes transform of the spectral distribution of $\mathcal{A}_{m}$ based on the signal-plus-noise sample covariance matrix.

- Step 2: based on the derived Stieltjes transform of $\mathcal{A}_{m}$ in Step 1, further consistently estimate the spectral distribution of ICV.

The two steps rely on two asymptotic results, Theorems 2.1 and 2.2, respectively. Roughly speaking, Theorem 2.1 enables us to make inferences about the signal sample covariance based on noisy observations, and Theorem 2.2 allows us to go further back to the population covariance matrix.

Approach II is more direct. It makes use of some special properties in the setting that we described in Section 1.1. The properties allow us to asymptotically eliminate the effect of noise, thus saving us from Step I above and enabling us to take only one step, which relies on Theorem 2.3. We also see below that Approach II is more robust, particularly in that it allows for rather general dependence structures in the noise process, both cross-sectional and temporal, and even dependence between the noise and price process. The drawback is that Approach II heavily relies on the setting in Section 1.1, while Approach I can be applied to wider situations involving noisy observations.

In the simulation studies, we explain in detail how to generalize the algorithm proposed by El Karoui (2008) to implement the estimation procedure in practice. We can see that Approaches I and II both yield satisfactory estimates of the spectral distribution of the targeting ICV matrix. Other algorithms, such as those introduced in Mestre (2008), Bai, Chen and Yao (2010) and Ledoit and Wolf (2015), can also be adapted to our setting.

The rest of the paper is organized as follows. Section 2 explains the two approaches and the underlying theories. Section 3 demonstrates how to implement the two approaches in practice. Section 4 concludes. The proofs are given in the supplementary article Xia and Zheng (2018).

Notation. For any $p \times p$ Hermitian matrix $\Sigma$ with eigenvalues $\lambda_{1}, \ldots, \lambda_{p}$, its empirical spectral distribution (ESD) is defined as

$$
F^{\Sigma}(x):=\frac{1}{p} \#\left\{j: \lambda_{j} \leq x\right\} \quad \text { for } x \in \mathbb{R} .
$$


The limit of ESD as $p \rightarrow \infty$, if it exists, is referred to as the limiting spectral distribution, or LSD for short; see, for example, the book Bai and Silverstein (2010). For any real matrix $\mathbf{A},\|\mathbf{A}\|=\sqrt{\lambda_{\max }\left(\mathbf{A A}^{T}\right)}$ denotes its spectral norm, where $\lambda_{\max }$ denotes the largest eigenvalue. For any nonnegative definite matrix $\mathbf{B}, \mathbf{B}^{1 / 2}$ denotes its square root matrix. For any $z \in \mathbb{C}$, write $\mathfrak{R}(z)$ and $\Im(z)$ as its real and imaginary parts, respectively, and $\bar{z}$ as its complex conjugate. For any distribution $F, m_{F}(\cdot)$ denotes its Stieltjes transform, which is defined as

$$
m_{F}(z)=\int \frac{1}{\lambda-z} d F(\lambda) \quad \text { for } z \in \mathbb{C}^{+}:=\{z \in \mathbb{C}: \Im(z)>0\} .
$$

In particular, the Stieltjes transform of $F^{\Sigma}$ above, denoted by $m_{\Sigma}(\cdot):=m_{F^{\Sigma}}(\cdot)$, is given by

$$
m_{\Sigma}(z)=\frac{\operatorname{tr}\left((\Sigma-z \mathbf{I})^{-1}\right)}{p} \quad \text { for } z \in \mathbb{C}^{+},
$$

where $\mathbf{I}$ is the identity matrix. Finally, for any vector $\mathbf{x},|\mathbf{x}|$ stands for its Euclidean norm.

\section{Main results.}

2.1. Preliminary: Pre-averaging method. The pre-averaging (PAV) method is introduced in Jacod et al. (2009), Podolskij and Vetter (2009), and Christensen, Kinnebrock and Podolskij (2010) to deal with microstructure noise. Other approaches include the two/multi-scales estimators [Zhang (2006, 2011), Zhang, Mykland and Aït Sahalia (2005)], realized kernel [Barndorff-Nielsen et al. (2008, 2011)], and quasi-maximum likelihood method [Aït Sahalia, Fan and Xiu (2010), Xiu (2010)]. We use a slight variant of the PAV approach in this work. First, choose a window length $k$. Then, group the intervals $[(i-1) / n, i / n]$ for $i=$ $1, \ldots, 2 k \cdot\lfloor n /(2 k)\rfloor$ into $m:=\lfloor n /(2 k)\rfloor$ pairs of nonoverlapping windows, each of width $(2 k) / n$, where $\lfloor\cdot\rfloor$ represents rounding down to the nearest integer. Introduce the following notation for any process $\mathbf{V}=\left(\mathbf{V}_{t}\right)_{t \geq 0}$ :

$$
\begin{aligned}
\Delta \mathbf{V}_{i} & =\mathbf{V}_{i / n}-\mathbf{V}_{(i-1) / n}, \quad \overline{\mathbf{V}}_{i}=\frac{1}{k} \sum_{j=0}^{k-1} \mathbf{V}_{((i-1) k+j) / n}, \quad \text { and } \\
\Delta \overline{\mathbf{V}}_{2 i} & =\overline{\mathbf{V}}_{2 i}-\overline{\mathbf{V}}_{2 i-1} .
\end{aligned}
$$

With such notation, the observed return based on the pre-averaged price becomes

$$
\Delta \overline{\mathbf{Y}}_{2 i}=\Delta \overline{\mathbf{X}}_{2 i}+\Delta \overline{\boldsymbol{\varepsilon}}_{2 i}
$$

One key observation is that if $k$ is chosen to be of order $\sqrt{n}$ [which is the order chosen in Jacod et al. (2009), Podolskij and Vetter (2009) and Christensen, Kinnebrock and Podolskij (2010)] then, in (2.2), the "signal" $\Delta \overline{\mathbf{X}}_{2 i}$ and "noise" 
$\Delta \overline{\boldsymbol{\varepsilon}}_{2 i}$ can be shown to be of the same order of magnitude. Observe that with such a chosen window width, the resulting number of windows is only of order $\sqrt{n}$; hence our statement earlier that the effective sample size is only $O(\sqrt{n})$ and consequently, even in the one-dimensional case, the optimal rate of convergence for estimating ICV is only $\sqrt{\sqrt{n}}=n^{1 / 4}$.

\subsection{Approach $I$.}

2.2.1. Step 1: From signal-plus-noise back to signal. Our starting point is the PAV matrix, which is defined as a multiple of the sample covariance matrix of $\Delta \overline{\mathbf{Y}}_{2 i}$, the returns based on the pre-averaged prices:

$$
\mathrm{PAV}:=3 \sum_{i=1}^{m}\left(\Delta \overline{\mathbf{Y}}_{2 i}\right)\left(\Delta \overline{\mathbf{Y}}_{2 i}\right)^{T}
$$

[Coefficient 3 is inherited from Jacod et al. (2009) and comes from the convergence (2.9) below.] This is slightly different from the estimator in Jacod et al. (2009), particularly in that there is no bias correction term involved. This is because (1) in the high-dimensional setting, even with the bias correction, the PAV is still inconsistent, just as in high-dimensional multivariate analysis the sample covariance matrix is inconsistent; and (2) our version of the PAV facilitates further analysis, which leads us all the way back to the target ICV.

The matrix PAV can be viewed as the sample covariance matrix based on observations $\Delta \overline{\mathbf{X}}_{2 i}+\Delta \overline{\boldsymbol{\varepsilon}}_{2 i}$, which model the situation of the information vector $\Delta \overline{\mathbf{X}}_{2 i}$ being contaminated by additive noise $\Delta \overline{\boldsymbol{\varepsilon}}_{2 i}$. Dozier and Silverstein (2007a) consider such signal-plus-noise sample covariance matrices as

$$
\mathbf{S}_{n}=\frac{1}{n}\left(\mathbf{A}_{n}+\sigma_{n} \mathbf{E}_{n}\right)\left(\mathbf{A}_{n}+\sigma_{n} \mathbf{E}_{n}\right)^{T},
$$

where $\mathbf{A}_{n}$ indicates a matrix consisting of signals, while $\mathbf{E}_{n}$, independent of $\left(\mathbf{A}_{n}\right)$, consists of i.i.d. noise. Let $\mathcal{A}_{n}:=\mathbf{A}_{n} \mathbf{A}_{n}^{T} / n$ be the signal sample covariance matrix. Under certain regularity conditions, the authors show that if $F^{\mathcal{A}_{n}}$ converges to a probability distribution $F^{\mathcal{A}}$, then so does $F^{\mathbf{S}_{n}}$. They further show that the LSD of $\mathbf{S}_{n}$ is determined by $F^{\mathcal{A}}$ in that its Stieltjes transform $m=m(z)$ uniquely solves the following equation:

$$
m=\int \frac{d F^{\mathcal{A}}(t)}{\frac{t}{1+\sigma^{2} y m}-\left(1+\sigma^{2} y m\right) z+\sigma^{2}(1-y)} \quad \text { for all } z \in \mathbb{C}^{+},
$$

where $\sigma^{2}$ and $y$ are given in Assumptions (A.ii) and (A.iv) below.

Our goal in this article, as in many other applications, is to make inferences about signals based on noisy observations; in this case, to make inferences about $\mathcal{A}_{n}$ based on $\mathbf{S}_{n}$. This motivates us to investigate the problem from a different 
angle than Dozier and Silverstein (2007a). Unlike (2.4), which states how the LSD of $\mathbf{S}_{n}$ depends on that of $\mathcal{A}_{n}$, we show how the LSD of $\mathcal{A}_{n}$ depends on that of $\mathbf{S}_{n}$; see equation (2.5) below. We further explain how such a relation enables us to consistently estimate the ESD of $\mathcal{A}_{n}$ based on $\mathbf{S}_{n}$.

The relation that we establish is essentially an inverse relation of (2.4). Inverting such relations is in general notoriously difficult. For example, the MarčenkoPastur equation, which is similar to equation (2.4) and describes how the LSD of the sample covariance matrix depends on that of the population covariance matrix, is established long time ago in Marčenko and Pastur (1967), but it was after more than forty years that researchers realized how the (unobservable) ESD of the population covariance matrix can be recovered based on the (observable) ESD of the sample covariance matrix [Bai, Chen and Yao (2010), El Karoui (2008), Ledoit and Wolf (2015), Mestre (2008) etc]. In particular, Mestre (2008) derived an inverse formula for estimating individual population eigenvalues, under the assumption that the population covariance matrix admits only finitely many distinct eigenvalues with known multiplicity. Our first result, Theorem 2.1 below, gives an inverse relation of (2.4) that allows the derivation of the ESD of $\mathcal{A}_{n}$ based on that of $\mathbf{S}_{n}$, under rather general assumptions.

We impose the following assumptions on the underlying matrices. Assumptions (A.i) and (A.iii) are from Dozier and Silverstein (2007a); in particular, (A.i) is about the convergence of the ESD of the signal sample covariance matrix. Assumption (A.ii) allows the variance of noise to depend on $n$ as in the case of PAV. Assumption (A.iv) is standard in the studies of random matrices.

(A.i) $\mathbf{A}_{n}$ is $p \times n$, independent of $\boldsymbol{\varepsilon}_{n}$, and with $\mathcal{A}_{n}=(1 / n) \mathbf{A}_{n} \mathbf{A}_{n}^{T}, F^{\mathcal{A}_{n}} \stackrel{\mathcal{D}}{\rightarrow}$ $F^{\mathcal{A}}$, where $F^{\mathcal{A}}$ is a probability distribution with the Stieltjes transform denoted by $m_{\mathcal{A}}(\cdot)$;

(A.ii) $\sigma_{n} \geq 0$ with $\lim _{n \rightarrow \infty} \sigma_{n}=\sigma \in[0, \infty)$;

(A.iii) $\mathbf{E}_{n}=\left(\epsilon_{i j}\right)$ is $p \times n$ with the entries $\epsilon_{i j}$ being i.i.d. and centered with unit variance; and

(A.iv) $n=n(p)$ with $y_{n}=p / n \rightarrow y>0$ as $p \rightarrow \infty$.

We now present our first result about how the LSD of $\mathcal{A}_{n}$ depends on that of $\mathbf{S}_{n}$.

THEOREM 2.1. Suppose that Assumptions (A.i)-(A.iv) hold. Then, almost surely, the ESD of $\mathbf{S}_{n}$ converges in distribution to a probability distribution $F$. Moreover, if $F$ is supported by a finite interval $[a, b]$ with $a>0$ and possibly has a point mass at 0 , then $F^{\mathcal{A}}$ can be identified as follows. For all $z \in \mathbb{C}^{+}$such that $m_{\mathcal{A}}(z) \in D_{\mathcal{A}}\left(y, \sigma^{2}\right):=\left\{\xi \in \mathbb{C}: z\left(1-y \sigma^{2} \xi\right)^{2}-\sigma^{2}(y-1)\left(1-y \sigma^{2} \xi\right) \in \mathbb{C}^{+}\right\}$, $m_{\mathcal{A}}(z)$ uniquely solves the following equation:

$$
m_{\mathcal{A}}(z)=\int \frac{d F(\tau)}{\frac{\tau}{1-y \sigma^{2} m_{\mathcal{A}}(z)}-z\left(1-y \sigma^{2} m_{\mathcal{A}}(z)\right)+\sigma^{2}(y-1)} .
$$


REMARK 2.1. The restriction on $m_{\mathcal{A}}(z)$ to be in $D_{\mathcal{A}}$ is such that the integral on the right-hand side of (2.5) is well defined. Note that because $m_{\mathcal{A}}(z) \rightarrow 0$ and $z m_{\mathcal{A}}(z) \rightarrow-1$ as $\Im(z) \rightarrow \infty, m_{\mathcal{A}}(z)$ does belong to $D_{\mathcal{A}}$ for all $z$ with $\Im(z)$ sufficiently large. Furthermore, by the uniqueness of analytic continuation, knowing the values of $m_{\mathcal{A}}(z)$ for $z$ with $\mathfrak{s}(z)$ sufficiently large is sufficient to determine $m_{\mathcal{A}}(z)$ for all $z \in \mathbb{C}^{+}$.

Let us explain how Theorem 2.1 can be used to make inferences about signals based on noisy observations.

(i) In practice, we observe noisy observations and can compute $\mathbf{S}_{n}$ and hence its ESD. We can then replace $F$ in (2.5) with $F^{\mathbf{S}_{n}}$ and solve for $m_{\mathcal{A}_{n}}(z)$. The empirical version of (2.5) can be solved numerically using, for example, the R package "rootSolve." The uniqueness of the solution to equation (2.5) is theoretically justified using analytic tools. For its empirical version, we prove that if $\widehat{m_{\mathcal{A}_{n}}(z)}$ solves the empirical version, then it is close to the true $m_{\mathcal{A}_{n}}$ [see see Appendix D in the supplementary article Xia and Zheng (2018)]. This property guarantees that even if the empirical version of (2.5) admits multiple solutions, they are all close to the true one. Consequently, because $m_{\mathcal{A}_{n}}(z)$ fully characterizes the ESD of $\mathcal{A}_{n}$, the estimated $m_{\mathcal{A}_{n}}(z)$ enables us to consistently estimate the ESD. In the simulation studies, we explain in detail how to implement this procedure in practice.

(ii) More importantly, to further estimate the ESD of the population covariance matrix, in the next step to be developed, we need $m_{\mathcal{A}_{n}}(z)$. Theorem 2.1 provides such a necessary input. This is an important outcome of establishing the inverse relation (2.5).

In practice, if we are only interested in estimating the spectral distribution of the population covariance matrix, then, because in the second step we only need $m_{\mathcal{A}_{n}}(z)$, there is actually no need to estimate the ESD of $\mathcal{A}_{n}$. In the simulation studies, we still include this part but only for the purpose of illustrating the application of Theorem 2.1 .

We now apply Theorem 2.1 to our PAV matrix. As mentioned in the summary in Section 1.2, in Step 1, we relate the PAV matrix to an intermediate matrix $\mathcal{A}_{m}$ defined as follows:

$$
\mathcal{A}_{m}:=3 \sum_{i=1}^{m} \Delta \overline{\mathbf{X}}_{2 i} \cdot\left(\Delta \overline{\mathbf{X}}_{2 i}\right)^{T}
$$

It differs from the PAV matrix in that it does not involve the noise and can be regarded as a signal sample covariance matrix. The assumptions under our setting analogous to (A.i)-(A.iv) for Theorem 2.1 are then as follows. 
(B.i) the ESD of $\mathcal{A}_{m}$ converges to a probability distribution $F^{\mathcal{A}}$ with the Stieltjes transform denoted by $m_{\mathcal{A}}(z)$;

(B.ii) the noise $\left(\boldsymbol{\varepsilon}_{i}\right)_{1 \leq i \leq n}$ are independent of $\left(\mathbf{X}_{t}\right)$ and are i.i.d. with zero mean and covariance matrix $\boldsymbol{\Sigma}_{e}=\sigma_{p}^{2} \mathbf{I}$ for some $\sigma_{p}>0$ and $\sigma_{p} \rightarrow \sigma_{e}>0$ as $p \rightarrow \infty$;

(B.iii) $k=\lfloor\theta \sqrt{n}\rfloor$ for some $\theta \in(0, \infty)$, and $m=\left\lfloor\frac{n}{2 k}\right\rfloor$ satisfies $\lim _{p \rightarrow \infty} p / m=y$.

We then have the following corollary as a direct consequence of Theorem 2.1 .

COROllary 2.1. Suppose that for all p, $\left(\mathbf{X}_{t}\right)$ is a p-dimensional process satisfying (1.1). Suppose also that Assumptions (B.i)-(B.iii) hold. Then, almost surely, the ESD of PAV defined in (2.3) converges to a probability distribution $F$. Moreover, if $F$ is supported by a finite interval $[a, b]$ with $a>0$ and possibly has a point mass at 0 , then $F^{\mathcal{A}}$ can be identified as follows. For all $z \in \mathbb{C}^{+}$ such that $m_{\mathcal{A}}(z) \in D_{\mathcal{A}}\left(y, 3 \theta^{-2} \sigma_{e}^{2}\right), m_{\mathcal{A}}(z)$ uniquely solves the following equation:

$$
m_{\mathcal{A}}(z)=\int \frac{d F(\tau)}{\frac{\tau}{1-3 y \theta^{-2} \sigma_{e}^{2} m_{\mathcal{A}}(z)}-z\left(1-3 y \theta^{-2} \sigma_{e}^{2} m_{\mathcal{A}}(z)\right)+3 \theta^{-2} \sigma_{e}^{2}(y-1)}
$$

REMARK 2.2. Although Corollary 2.1 is stated for the case when noise components have the same standard deviations, it can readily be applied to the case when the covariance matrix $\Sigma_{e}$ is a general diagonal matrix, say, $\operatorname{diag}\left(d_{1}^{2}, \ldots, d_{p}^{2}\right)$. To see this, let $d_{\max }^{2}=\max \left(d_{1}^{2}, \ldots, d_{p}^{2}\right)$. We can then artificially add additional $\tilde{\boldsymbol{\varepsilon}}_{i}$ to the original observations, where $\tilde{\boldsymbol{\varepsilon}}_{i}$ are independent of $\boldsymbol{\varepsilon}_{i}$ and are i.i.d. with zero mean and covariance matrix $\widetilde{\boldsymbol{\Sigma}}_{e}=\operatorname{diag}\left(d_{\max }^{2}-d_{1}^{2}, \ldots, d_{\max }^{2}-d_{p}^{2}\right)$. The noise components in the modified observations then have the same standard deviation $d_{\max }$, and Corollary 2.1 can be applied. Note that the variances, $d_{1}^{2}, \ldots, d_{p}^{2}$, can be consistently estimated; see, for example, Theorem A.1 in Zhang, Mykland and Aït Sahalia (2005). A similar remark applies to Theorem 2.1 .

2.2.2. Step 2: From signal to population. Step 1 enables us to infer the spectral distribution of $\mathcal{A}_{m}$ based on PAV. However, just as in high-dimensional multivariate analysis where the sample covariance matrix is not consistent, neither is $\mathcal{A}_{m}$. This is why we need this second step, which enables us to go further back to the ICV matrix.

First, we introduce some structural assumptions on the latent process $\mathbf{X}$ in order to go further. Note that the term $\Delta \overline{\mathbf{V}}_{2 i}$ in (2.1) can be written in a more clear form 
by using the triangular kernel:

$$
\begin{aligned}
\Delta \overline{\mathbf{V}}_{2 i} & =\frac{1}{k} \sum_{j=0}^{k-1}\left(\mathbf{V}_{((2 i-1) k+j) / n}-\mathbf{V}_{((2 i-2) k+j) / n}\right) \\
& =\frac{1}{k} \sum_{j=0}^{k-1} \sum_{\ell=1}^{k} \Delta \mathbf{V}_{(2 i-2) k+j+\ell} \\
& =\sum_{|j|<k}\left(1-\frac{|j|}{k}\right) \Delta \mathbf{V}_{(2 i-1) k+j} .
\end{aligned}
$$

Based on this, it can be shown that if the dimension $p$ is fixed, then, as $n \rightarrow \infty$,

$$
\sum_{i=1}^{m} \Delta \overline{\mathbf{X}}_{2 i} \cdot\left(\Delta \overline{\mathbf{X}}_{2 i}\right)^{T} \stackrel{p}{\longrightarrow} \frac{\mathrm{ICV}}{3} \quad \text { hence } \mathcal{A}_{m} \stackrel{p}{\longrightarrow} \text { ICV }
$$

It is also easy to verify that

$$
\Delta \overline{\boldsymbol{\varepsilon}}_{2 i} \stackrel{\mathcal{D}}{=} \sqrt{\frac{2}{k}} \mathbf{e}_{i}
$$

where $\mathbf{e}_{i}$ s are i.i.d. random vectors with mean zero and covariance matrix $\boldsymbol{\Sigma}_{e}$.

Corollary 2.1 in Step 1 allows us to consistently estimate the ESD of $\mathcal{A}_{m}$. In light of the convergence (2.9), it would have been sufficient for us to make inferences about the ICV if the convergence (2.9) also held in the high-dimensional case. Unfortunately, this is not the case, and a further step to go from $\mathcal{A}_{m}$ to ICV is needed. Such an inference is generally impossible, as can be seen in the following. ICV is an integral $\int_{0}^{1} \boldsymbol{\Theta}_{t} \boldsymbol{\Theta}_{t}^{T} d t$. In the simple situation where $\boldsymbol{\mu}_{t} \equiv 0$ and $\boldsymbol{\Theta}_{t}$ is deterministic, the building blocks in defining $\mathcal{A}_{m}, \Delta \mathbf{X}_{i}$, are multivariate normals with mean 0 and covariance matrices $\int_{(i-1) / n}^{i / n} \boldsymbol{\Theta}_{t} \boldsymbol{\Theta}_{t}^{T} d t$. The bottom line is all the $n$ covariance matrices, $\int_{(i-1) / n}^{i / n} \boldsymbol{\Theta}_{t} \boldsymbol{\Theta}_{t}^{T} d t$ for $i=1, \ldots, n$, could be very different from the ICV! We can easily change the $n$ covariance matrices $\int_{(i-1) / n}^{i / n} \boldsymbol{\Theta}_{t} \boldsymbol{\Theta}_{t}^{T} d t$ and hence the distributions of $\Delta \mathbf{X}_{i}$ without changing ICV. And as both the dimension $p$ and observation frequency $n$ go to infinity, there is too much freedom in the underlying distributions, which makes inferences about ICV impossible. Certain structural assumptions are necessary to turn the impossible possible. The simplest is to assume that $\boldsymbol{\Theta}_{t} \equiv \boldsymbol{\Theta}$, in which case $\Delta \mathbf{X}_{i}$ are i.i.d. The apparent drawback of this assumption is that it cannot capture stochastic volatility, which is a stylized feature in financial data. The following class of processes, introduced in Zheng and Li (2011), accommodates both stochastic volatility and the leverage effect while still making the inference about ICV possible (and the theory is already much more complicated than the i.i.d. observation setting). 
Definition 2.1. Suppose that $\left(\mathbf{X}_{t}\right)$ is a $p$-dimensional process satisfying (1.1). We say that $\left(\mathbf{X}_{t}\right)$ belongs to Class $\mathcal{C}$ if, almost surely, there exist $\left(\gamma_{t}\right) \in$ $D([0,1] ; \mathbb{R})$ and $\boldsymbol{\Lambda}$ a $p \times p$ matrix satisfying $\operatorname{tr}\left(\boldsymbol{\Lambda} \mathbf{\Lambda}^{T}\right)=p$ such that

$$
\boldsymbol{\Theta}_{t}=\gamma_{t} \boldsymbol{\Lambda}
$$

where $D([0,1] ; \mathbb{R})$ stands for the space of càdlàg functions from $[0,1]$ to $\mathbb{R}$.

REMARK 2.3. The convention that $\operatorname{tr}\left(\boldsymbol{\Lambda} \boldsymbol{\Lambda}^{T}\right)=p$ is made to resolve the nonidentifiability built into the formulation (2.10), in which one can multiply $\left(\gamma_{t}\right)$ and divide $\boldsymbol{\Lambda}$ by a same constant without modifying the process $\left(\boldsymbol{\Theta}_{t}\right)$. It is thus not a restriction.

Class $\mathcal{C}$ incorporates some widely used models as special cases:

- The simplest case is when the drift $\boldsymbol{\mu}_{t} \equiv 0$ and $\gamma_{t} \equiv \gamma$, in which case the returns $\Delta \mathbf{X}_{i}$ are i.i.d. $N\left(0, \gamma^{2} / n \cdot \boldsymbol{\Lambda} \mathbf{\Lambda}^{T}\right)$.

- More generally, again, when the drift $\boldsymbol{\mu}_{t} \equiv 0$ while $\left(\gamma_{t}\right)$ is independent of the underlying Brownian motion $\left(\mathbf{W}_{t}\right)$, the returns $\Delta \mathbf{X}_{i}$ follow mixed normal distributions.

- Mixed normal distributions, or their asymptotic equivalent form in the highdimensional setting, elliptic distributions [see Section 2 of El Karoui (2013) for the asymptotic equivalence], have been widely used in financial applications. McNeil, Frey and Embrechts (2005) state that "elliptical distributions ... provided far superior models to the multivariate normal for daily and weekly US stock-return data" and that "multivariate return data for groups of returns of a similar type often look roughly elliptical."

- More recently, El Karoui, in a series of papers [El Karoui (2009, 2010a, 2013)], studied the Markowitz optimization problem under the setting that the returns follow mixed normal/elliptic distributions.

- Furthermore, Class $\mathcal{C}$ allows the drift $\left(\boldsymbol{\mu}_{t}\right)$ to be nonzero and more importantly, the $\left(\gamma_{t}\right)$ process to be stochastic and even dependent on the Brownian motion $\left(\mathbf{W}_{t}\right)$ that drives the price process, thus featuring the so-called leverage effect in financial econometrics. The leverage effect is an important stylized fact of financial returns and has drawn a great deal of attention in recent years; see, for example, Aït-Sahalia, Fan and Li (2010) and Wang and Mykland (2014).

Observe that if $\left(\mathbf{X}_{t}\right)$ belongs to Class $\mathcal{C}$, then the ICV matrix

$$
\mathrm{ICV}=\int_{0}^{1} \gamma_{t}^{2} d t \cdot \breve{\Sigma} \quad \text { where } \breve{\Sigma}=\mathbf{\Lambda} \mathbf{\Lambda}^{T} .
$$

Furthermore, if the drift process $\boldsymbol{\mu}_{t} \equiv 0$ and $\left(\gamma_{t}\right)$ is independent of $\left(\mathbf{W}_{t}\right)$, then, conditional on $\left(\gamma_{t}\right)$ and using (2.8), we have

$$
\Delta \overline{\mathbf{X}}_{2 i} \stackrel{\mathcal{D}}{=} \sqrt{w_{i}} \breve{\Sigma}^{1 / 2} \mathbf{Z}_{i},
$$


where $\mathbf{Z}_{i}=\left(Z_{i}^{1}, \ldots, Z_{i}^{p}\right)^{T}$ consists of independent standard normals and

$$
w_{i}=\sum_{|j|<k}\left(1-\frac{|j|}{k}\right)^{2} \int_{\frac{(2 i-1) k+j-1}{n}}^{\frac{(2 i-1) k+j}{n}} \gamma_{t}^{2} d t .
$$

It follows that

$$
\mathcal{A}_{m}=3 \sum_{i=1}^{m} \Delta \overline{\mathbf{X}}_{2 i} \cdot\left(\Delta \overline{\mathbf{X}}_{2 i}\right)^{T} \stackrel{\mathcal{D}}{=} 3 \sum_{i=1}^{m} w_{i} \breve{\mathbf{\Sigma}}^{1 / 2} \mathbf{Z}_{i} \mathbf{Z}_{i}^{T} \breve{\mathbf{\Sigma}}^{1 / 2}
$$

We now explain how to make further inferences about the ICV matrix based on $\mathcal{A}_{m}$. Doing so relies on another asymptotic result which relates $\mathcal{A}_{m}$ to ICV.

We impose the following assumptions on the underlying process. They are inherited from Proposition 5 of Zheng and Li (2011), and we refer the readers to that article for further background and explanations. Observe in particular that Assumption (C.v) allows the covolatility process to be dependent on the Brownian motion that drives the price processes. Such dependence allows us to capture the leverage effect. Assumptions (C.iv) and (C.vi) concern the spectral norm of the ICV matrix. We do not require the norm to be bounded, which allows, for example, spike eigenvalues.

\section{Assumption C.}

(C.i) For all $p,\left(\mathbf{X}_{t}\right)$ is a $p$-dimensional process in Class $\mathcal{C}$ for some drift process $\boldsymbol{\mu}_{t}=\left(\mu_{t}^{1}, \ldots, \mu_{t}^{p}\right)^{T}$ and covolatility process $\left(\boldsymbol{\Theta}_{t}\right)=\left(\gamma_{t} \boldsymbol{\Lambda}\right)$;

(C.ii) there exists $C_{0}<\infty$ such that for all $p$ and all $j=1, \ldots, p,\left|\mu_{t}^{j}\right| \leq C_{0}$ for all $t \in[0,1)$ almost surely;

(C.iii) as $p \rightarrow \infty$, the ESD of $\breve{\Sigma}=\boldsymbol{\Lambda} \boldsymbol{\Lambda}^{T}$ converges to a probability distribution $\breve{H}$;

(C.iv) there exist $C_{1}<\infty$ and $\kappa<1 / 6$ such that for all $p,\|\breve{\Sigma}\| \leq C_{1} p^{\kappa}$ almost surely;

(C.v) there exists a sequence of index sets $\mathcal{I}_{p}$ satisfying $\mathcal{I}_{p} \subset\{1, \ldots, p\}$ and $\# \mathcal{I}_{p}=o(p)$ such that $\left(\gamma_{t}\right)$ may depend on $\left(\mathbf{W}_{t}\right)$ but only on $\left(W_{t}^{j}: j \in \mathcal{I}_{p}\right)$;

(C.vi) there exists $C_{2}<\infty$ such that for all $p$ and for all $t \in[0,1),\left|\gamma_{t}\right| \leq$ $C_{2}$ almost surely, and additionally, almost surely, $\left(\gamma_{t}\right)$ converges uniformly to a nonzero process $\left(\gamma_{t}^{*}\right)$ that is piecewise continuous with finitely many jumps.

We then have the following result connecting $\mathcal{A}_{m}$ with ICV.

THEOREM 2.2. Suppose that Assumptions (C.i)-(C.vi) and (B.iii) hold, then as $p \rightarrow \infty$ :

(i) the ESDs of ICV and $\mathcal{A}_{m}$ converge to probability distributions $H$ and $F^{\mathcal{A}}$ respectively, where

$$
H(x)=\breve{H}(x / \zeta) \quad \text { for all } x \geq 0 \text { with } \zeta=\int_{0}^{1}\left(\gamma_{t}^{*}\right)^{2} d t
$$


(ii) $F^{\mathcal{A}}$ and $H$ are related as follows:

$$
m_{\mathcal{A}}(z)=-\frac{1}{z} \int \frac{\zeta}{\tau M(z)+\zeta} d H(\tau)
$$

where $M(z)$ and another function $\tilde{m}(z)$ uniquely solve the following equations in $\mathbb{C}^{+} \times \mathbb{C}^{+}$:

$$
\left\{\begin{array}{l}
M(z)=-\frac{1}{z} \int_{0}^{1} \frac{\left(\gamma_{s}^{*}\right)^{2}}{1+y \tilde{m}(z)\left(\gamma_{s}^{*}\right)^{2}} d s \\
\tilde{m}(z)=-\frac{1}{z} \int \frac{\tau}{\tau M(z)+\zeta} d H(\tau)
\end{array}\right.
$$

Equation (2.14) in Theorem 2.2 forms the basis for us to further estimate the ESD of ICV. It involves an unknown function $M(z)$, which can be solved as follows. First, note that multiplying $\tilde{m}(z)$ and $M(z)$ on both sides of the first and second equations in (2.15), respectively, yields

$$
\left\{\begin{array}{l}
M(z) \cdot \tilde{m}(z)=-\frac{1}{y z}+\frac{1}{y z} \int_{0}^{1} \frac{1}{1+y \tilde{m}(z)\left(\gamma_{s}^{*}\right)^{2}} d s \\
M(z) \cdot \tilde{m}(z)=-\frac{1}{z} \int \frac{\tau M(z)}{\tau M(z)+\zeta} d H(\tau)=-\frac{1}{z}-m_{\mathcal{A}}(z),
\end{array}\right.
$$

where the last step is due to (2.14). It follows that

$$
-\frac{1}{z}-m_{\mathcal{A}}(z)=-\frac{1}{y z}+\frac{1}{y z} \int_{0}^{1} \frac{1}{1+y \tilde{m}(z)\left(\gamma_{s}^{*}\right)^{2}} d s,
$$

and $\tilde{m}(z)=-\left(1 / z+m_{\mathcal{A}}(z)\right) / M(z)$. Substituting the last expression of $\tilde{m}(z)$ into equation (2.16), yields

$$
\int_{0}^{1} \frac{M(z)}{M(z)-\left(\gamma_{s}^{*}\right)^{2} y\left(z^{-1}+m_{\mathcal{A}}(z)\right)} d s=1-y-y z m_{\mathcal{A}}(z) .
$$

$M(z)$ is then obtained by plugging in the $m_{\mathcal{A}}(z)$ that we derived in Step 1 into (2.17) and solving for the solution that is unique in $\mathbb{C}^{+}$by Theorem 1 in Zheng and $\mathrm{Li}$ (2011).

Having solved $M(z)$, we can then utilize equation (2.14) to estimate the ESD of ICV by generalizing the algorithms in El Karoui (2008), Mestre (2008), Bai, Chen and Yao (2010) and Ledoit and Wolf (2015), etc. The resulting estimate can be shown to be consistent by using an argument similar to that for Theorem 2 of El Karoui (2008). The estimation procedure is explained in detail in the simulation studies. 
2.3. Approach II. The second step in Approach I involves the process $\left(\gamma_{s}^{*}\right)$ which is unknown in practice. Estimating this process inevitably introduces an additional source of error. Motivated by this consideration, we draw ideas from Zheng and Li (2011) and develop an alternative approach that overcomes this difficulty. It is also worth mentioning that the alternative approach allows for rather general dependence structures in the noise process, both cross-sectional and temporal, and even dependence between the noise and price process. The temporal dependence between microstructure noise has been documented in recent studies; see, for example, Hansen and Lunde (2006), Ubukata and Oya (2009) and Jacod, Li and Zheng (2017).

We shall define a matrix that is an extension of the time-variation adjusted RCV matrix introduced in Zheng and Li (2011) to our noisy setting. To start, fix an $\alpha \in(1 / 2,1)$ and $\theta \in(0, \infty)$, and let $k=\left\lfloor\theta n^{\alpha}\right\rfloor$ and $m=\lfloor n /(2 k)\rfloor$. The timevariation adjusted PAV matrix is then defined as

$$
\mathcal{B}_{m}:=3 \frac{\sum_{i=1}^{m}\left|\Delta \overline{\mathbf{Y}}_{2 i}\right|^{2}}{m} \cdot \sum_{i=1}^{m} \frac{\Delta \overline{\mathbf{Y}}_{2 i}\left(\Delta \overline{\mathbf{Y}}_{2 i}\right)^{T}}{\left|\Delta \overline{\mathbf{Y}}_{2 i}\right|^{2}}=3 \frac{\sum_{i=1}^{m}\left|\Delta \overline{\mathbf{Y}}_{2 i}\right|^{2}}{p} \widetilde{\mathbf{\Sigma}},
$$

where

$$
\widetilde{\Sigma}:=\frac{p}{m} \sum_{i=1}^{m} \frac{\Delta \overline{\mathbf{Y}}_{2 i}\left(\Delta \overline{\mathbf{Y}}_{2 i}\right)^{T}}{\left|\Delta \overline{\mathbf{Y}}_{2 i}\right|^{2}}
$$

Note that here the window length $k$ has a higher order than in Theorem 2.2. The reason is that, after pre-averaging, the underlying returns are $O_{p}(\sqrt{k / n})$ and the noises are $O_{p}(\sqrt{1 / k})$. In Theorem 2.2, we balance the orders of the two terms by choosing $k=O(\sqrt{n})$; here we take $k=O\left(n^{\alpha}\right)$ for some $\alpha>1 / 2$, which enables us to asymptotically eliminate the effect of noise.

Next, recall the concept of $\rho$-mixing coefficients.

Definition 2.2. Suppose that $U=\left(U_{k}, k \in \mathbb{Z}\right)$ is a stationary time series. For $-\infty \leq j \leq \ell \leq \infty$, let $\mathcal{F}_{j}^{\ell}$ be the $\sigma$-field generated by the random variables $\left(U_{k}: j \leq k \leq \ell\right)$. The $\rho$-mixing coefficients are defined as

$$
\rho(r)=\sup _{f \in \mathcal{L}^{2}\left(\mathcal{F}_{-\infty}^{0}\right), g \in \mathcal{L}^{2}\left(\mathcal{F}_{r}^{\infty}\right)}|\operatorname{Corr}(f, g)| \quad \text { for } r \in \mathbb{N},
$$

where, for any probability space $\Omega, \mathcal{L}^{2}(\Omega)$ refers to the space of square-integrable, $\Omega$-measurable random variables.

We now introduce a number of assumptions. Assumption (D.i) below says that we allow for rather general dependence structures in the noise process, both cross-sectional and temporal. We actually do not put any restrictions on the crosssectional dependence, and even dependence between the noise and price process is allowed. Note also that Jacod, Li and Zheng (2017) provides an approach to 
estimate the decay rate of the $\rho$-mixing coefficients. Assumption (D.ii) concerns the dependence between the covolatility process and the Brownian motion that drives the price processes. Assumption (D.iv) is about the boundedness of individual volatilities.

(D.i) For all $j=1, \ldots, p$, the noise $\left(\varepsilon_{i}^{j}\right)$ is stationary, has mean 0 and bounded $4 \ell$ th moments, and has $\rho$-mixing coefficients $\rho^{j}(r)$ satisfying $\max _{j=1, \ldots, p} \rho^{j}(r)=O\left(r^{-\ell}\right)$ for some integer $\ell \geq 2$;

(D.ii) there exist $0 \leq \delta_{1}<1 / 2$ and a sequence of index sets $\mathcal{I}_{p}$ satisfying $\mathcal{I}_{p} \subset\{1, \ldots, p\}$ and $\# \mathcal{I}_{p}=O\left(p^{\delta_{1}}\right)$ such that $\left(\gamma_{t}\right)$ may depend on $\left(\mathbf{W}_{t}\right)$ but only on $\left(W_{t}^{j}: j \in \mathcal{I}_{p}\right)$;

(D.iii) there exists $C_{1}<\infty$ such that for all $p,\left|\gamma_{t}\right| \in\left(1 / C_{1}, C_{1}\right)$ for all $t \in$ $[0,1)$ almost surely;

(D.iv) there exists $C_{2}<\infty$ such that for all $p$ and all $j$, the individual volatilities $\sigma_{t}=\sqrt{\left(\gamma_{t}\right)^{2} \cdot \sum_{k=1}^{p}\left(\Lambda_{j k}\right)^{2}} \in\left(1 / C_{2}, C_{2}\right)$ for all $t \in[0,1]$ almost surely;

(D.v) there exist $C_{3}<\infty$ and $0 \leq \delta_{2}<1 / 2$ such that for all $p,\|\mathrm{ICV}\| \leq$ $C_{3} p^{\delta_{2}}$ almost surely;

(D.vi) the $\delta_{1}$ in (D.ii) and $\delta_{2}$ in (D.v) satisfy that $\delta_{1}+\delta_{2}<1 / 2$;

(D.vii) $k=\left\lfloor\theta n^{\alpha}\right\rfloor$ for some $\theta \in(0, \infty)$ and $\alpha \in[(3+\ell) /(2 \ell+2), 1)$, and $m=$ $\left\lfloor\frac{n}{2 k}\right\rfloor$ satisfy $\lim _{p \rightarrow \infty} p / m=y>0$, where $\ell$ is the integer in (D.i).

REMARK 2.4. Careful readers may have noted that Assumptions (B.iii) and (D.vii) are mathematically incompatible, as Assumption (B.iii) requires $p=$ $O(\sqrt{n})$ while Assumption (D.vii) requires $p=O\left(n^{1-\alpha}\right)$ for some $\alpha \in(1 / 2,1)$. The two assumptions are, however, perfectly compatible in practice when we deal with finite samples. Take the choices of $(p, n, k)$ in the simulation study in Section 3.1 below, for example. There, we take $(p, n)=(100,23,400)$. When applying Corollary 2.1 and Theorem 2.2, we take $k=\lfloor 0.5 \sqrt{n}\rfloor=76$, which leads to $y=p /\lfloor n / 2 k\rfloor \approx 0.7$ in Assumption (B.iii); when applying Theorem 2.3 below, we take $k=\left\lfloor 1.5 n^{0.6}\right\rfloor=627$, which gives $y=p /\lfloor n / 2 k\rfloor \approx 5.6$ in Assumption (D.vii).

We have the following convergence result connecting $\mathcal{B}_{m}$ with ICV.

Theorem 2.3. Suppose that Assumptions (C.i), (C.ii), (C.iii), (C.vi), and (D.i)-(D.vii) hold. Then, as $p \rightarrow \infty$, the ESDs of ICV and $\mathcal{B}_{m}$ converge almost surely to probability distributions $H$ and $F^{\mathcal{B}}$, respectively, where $H$ satisfies (2.13) and $F^{\mathcal{B}}$ is determined by $H$ in that its Stieltjes transform, denoted by $m_{\mathcal{B}}(z)$, satisfies the following (standard) Marčenko-Pastur equation:

$$
m_{\mathcal{B}}(z)=\int_{\tau \in \mathbb{R}} \frac{1}{\tau\left(1-y\left(1+z m_{\mathcal{B}}(z)\right)\right)-z} d H(\tau) \quad \text { for } z \in \mathbb{C}^{+} .
$$

Theorem 2.3 states that the LSDs of ICV and $\mathcal{B}_{m}$ are related via the MarčenkoPastur equation. Several algorithms have been developed to consistently recover 
$H$ by inverting the Marčenko-Pastur equation; see, for example, Bai, Chen and Yao (2010), El Karoui (2008), Ledoit and Wolf (2015), Mestre (2008) etc. We can therefore consistently estimate the ESD of ICV by using these existing algorithms.

2.4. A remark about asynchronicity. In multivariate high-frequency data analysis, in addition to microstructure noise, there is another challenge due to asynchronous trading. In practice, different stocks are traded at different times; consequently, the tick-by-tick data are not observed synchronously. There are several existing methods for synchronizing data, such as the refresh times [BarndorffNielsen et al. (2011)] and previous tick methods [Zhang (2011)]. Asynchronicity is less of an issue than microstructure noise. For example, as pointed out in Zhang (2011), asynchronicity does not induce bias in the two-scales estimator, and even the asymptotic variance is the same as if there is no asynchronicity. While a rigorous treatment is beyond the scope of this article, we expect our methods to work for asynchronous data as well. The reason, roughly speaking, is as follows. Take the previous tick method for example. Here, we choose a (usually equally spaced) grid of time points $0=t_{0}<t_{1}<\cdots<t_{n}=1$, and for each stock $j$, for each time point $t_{i}$, let $\tau_{i}^{j}$ be the latest transaction time before $t_{i}$. One then acts as if one observes $Y_{\tau_{i}^{j}}^{j}$ at time $t_{i}$ for stock $j$. With the original additive model at time $\tau_{i}^{j}$ :

$$
Y_{\tau_{i}^{j}}^{j}=X_{\tau_{i}^{j}}^{j}+\varepsilon_{i}^{j}
$$

we have at time $t_{i}$,

$$
Y_{t_{i}}^{j}:=Y_{\tau_{i}^{j}}^{j}=X_{t_{i}}^{j}+\left(\left(X_{\tau_{i}^{j}}^{j}-X_{t_{i}}^{j}\right)+\varepsilon_{i}^{j}\right)
$$

In other words, the asynchronicity induces an additional error $\left(X_{\tau_{i}^{j}}^{j}-X_{t_{i}}^{j}\right)$. The error is, however, diminishingly small as the sampling frequency $n \rightarrow \infty$ because $X_{\tau_{i}^{j}}^{j}-X_{t_{i}}^{j}=O_{p}\left(\sqrt{t_{i}-\tau_{i}^{j}}\right)=o_{p}(1)$. In short, asynchronicity induces an additional error (and violates our model assumption); fortunately, the error is of negligible order compared with the microstructure noise $\left(\varepsilon_{i}^{j}\right)$. We therefore keep our focus on the model (1.3). In simulation studies, in addition to the synchronous observation setting, we consider an asynchronous setting where the observation times for different stocks are independent Poisson processes. We shall see that our methods still work well (see Section 3.2 for more details).

3. Simulation studies. In this section, we demonstrate how to estimate the ESD of ICV by using Approach I, which uses the PAV matrix, and Approach II, which uses the alternative matrix $\mathcal{B}_{m}$. 
3.1. When observations are synchronous. We first consider a setting where observations are synchronous. To generate the underlying process $\mathbf{X}$, the process $\left(\gamma_{t}\right)$ in Definition 2.1 is taken to be a stochastic U-shaped $\left(\gamma_{t}\right)$ process as follows:

$$
d \gamma_{t}=-\rho\left(\gamma_{t}-\phi_{t}\right) d t+\sigma d \widetilde{W}_{t} \quad \text { for } t \in[0,1],
$$

where $\rho=10, \sigma=0.05$,

$$
\phi_{t}=2 \sqrt{0.0009+0.0008 \cos (2 \pi t)},
$$

and $\widetilde{W}_{t}=\sum_{i=1}^{p} W_{t}^{i} / \sqrt{p}$ with $W_{t}^{i}$ being the $i$ th component of the Brownian motion $\left(\mathbf{W}_{t}\right)$ that drives the price process. Observe that such a formulation makes $\left(\gamma_{t}\right)$ dependent on all the component of the underlying Brownian motion; hence, Assumptions (C.v) and (D.ii) are both violated. However, we shall see that our methods still work well. A sample path of $\left(\gamma_{t}\right)$ is given in Figure 7 in the supplementary article of Xia and Zheng (2018).

Next, the matrix $\breve{\Sigma}=\Lambda \Lambda^{T}$ is taken to be $\mathbf{U D U} \mathbf{U}^{\mathbf{T}}$, where $\mathbf{U}$ is a random orthogonal matrix and $\mathbf{D}$ is a diagonal matrix whose diagonal entries are drawn independently from the Beta $(1,3)$ distribution. Such generated $\breve{\Sigma}$ does not necessarily have trace $p$, but as we pointed out in Remark 2.3 , the assumption $\operatorname{tr}(\breve{\Sigma})=p$ is a convention rather than a requirement. With such $\left(\gamma_{t}\right)$ and $\breve{\Sigma}$, the individual daily volatilities are around 3\%, which is similar to what one observes in practice. The latent $\log$ price process $\left(\mathbf{X}_{t}\right)$ follows:

$$
d \mathbf{X}_{t}=\gamma_{t} \mathbf{\Lambda} d \mathbf{W}_{t} \quad \text { where } \boldsymbol{\Lambda}=\breve{\mathbf{\Sigma}}^{1 / 2}
$$

Finally, the noise $\left(\boldsymbol{\varepsilon}_{i}\right)_{1 \leq i \leq n}$ is taken to be i.i.d. $N(0,0.0002 \mathbf{I})$.

In the studies below, the dimension, that is, the number of stocks $p$, is taken to be 100 , and the observation frequency $n$ is set to be 23,400, which corresponds to one observation per second on a regular trading day. Note again that because of the presence of noise, the "effective sample size" is only of order $\sqrt{n} \approx 153$, which is comparable to our chosen $p=100$.

3.1.1. Estimation using Approach I: Synchronous setting. We start with Approach I, which involves two steps.

In the first step, we replace $F$ in equation (2.7) with the ESD of PAV and solve for $m_{\mathcal{A}}(z)$ using the R package "rootSolve." The window length $k$ in defining PAV is set to be $\lfloor 0.5 \sqrt{n}\rfloor=76$. As to the $m_{\mathcal{A}}(z)$ to be solved, we choose a set of $z$ 's whose real and imaginary parts are equally spaced in the intervals $[-20,0]$ and $[1,20]$, respectively. Denote these $z$ 's by $\left\{z_{j}\right\}_{j=1}^{J}$ and the estimated $m_{\mathcal{A}_{m}}\left(z_{j}\right)$ by $\left.\widehat{m_{\mathcal{A}_{m}}\left(z_{j}\right.}\right)$. We then need to estimate the ESD of $\mathcal{A}_{m}$ based on $\left.\left\{\widehat{m_{\mathcal{A}_{m}}\left(z_{j}\right.}\right)\right\}_{j=1}^{J}$, which we do as follows.

Inspired by the nonparametric estimation method proposed in El Karoui (2008), we approximate $F^{\mathcal{A}_{m}}$ with a weighted sum of point masses

$$
F^{\mathcal{A}_{m}} \approx \sum_{k=1}^{K} w_{k} \delta_{x_{k}},
$$


where $\left\{x_{1}<x_{2}<\cdots<x_{K}\right\}$ is a grid of points to be specified and the $w_{k}$ s are weights to be estimated. To choose the grid $\left\{x_{k}\right\}_{k=1}^{K}$, naturally, we would like $\left[x_{1}, x_{K}\right]$ to cover the support of $F^{\mathcal{A}_{m}}$, which is unknown. To overcome this difficulty, note that the support of the ESD of PAV always covers that of $\mathcal{A}_{m}$; hence, we can choose $x_{k}$ s to be equally spaced between 0 and the largest eigenvalue of PAV, and we are guaranteed that $\left[x_{1}, x_{K}\right]$ covers the support of $F^{\mathcal{A}_{m}}$.

Next, we discuss how to estimate the weights $\left\{w_{k}\right\}$ in (3.2). Observe that the discretization (3.2) gives an approximate Stieltjes transform of $F^{\mathcal{A}_{m}}$ as $\sum_{k=1}^{K} \frac{w_{k}}{x_{k}-z}$. Let

$$
\left.e_{j}^{\prime}:=\widehat{m_{\mathcal{A}_{m}}\left(z_{j}\right.}\right)-\sum_{k=1}^{K} \frac{w_{k}}{x_{k}-z_{j}}, \quad j=1, \ldots, J
$$

be the approximation errors. The weights $\left\{w_{k}\right\}_{k=1}^{K}$ are then estimated by minimizing the approximation errors:

$$
\underset{\left(w_{1}, \ldots, w_{k}\right)}{\arg \min } \max _{j=1,2, \ldots, J} \max \left\{\left|\Re\left(e_{j}\right)\right|,\left|\mathfrak{I}\left(e_{j}\right)\right|\right\}
$$

$$
\text { subject to } \sum_{k=1}^{K} w_{k}=1 \quad \text { and } \quad w_{k} \geq 0 .
$$

Next, in Step 2, we estimate the ESD of ICV. By plugging in the $\left.\left\{\widehat{m_{\mathcal{A}_{m}}\left(z_{j}\right.}\right)\right\}_{j=1}^{J}$ obtained in the first step and solving equation (2.17), we obtain $\left\{\widehat{M\left(z_{j}\right)}\right\}_{j=1}^{J}$. The estimation of the ESD of ICV is then conducted similarly as above as follows. Discretize the ESD of ICV as

$$
F^{\mathrm{ICV}} \approx \sum_{k=1}^{K} c_{k} \delta_{x_{k}}
$$

where $c_{k}$ s are again weights to be estimated. By equation (2.14), we expect that

$$
e_{j}^{\prime \prime}:=\widehat{m_{\mathcal{A}_{m}}\left(z_{j}\right)}+\frac{1}{z_{j}} \cdot \sum_{k=1}^{K} c_{k} \frac{\zeta}{x_{k} \widehat{M\left(z_{j}\right)}+\zeta}
$$

to be small. The $c_{k} \mathrm{~s}$ are then estimated by minimizing the approximation errors $e_{j}^{\prime \prime}$ just as in (3.3).

Figures 1 and 2 below illustrate the estimation results. The left plot of Figure 1 shows three ESDs, those of ICV, $\mathcal{A}_{m}$, and PAV. The three curves are clearly different from each: the difference between PAV and $\mathcal{A}_{m}$ is induced by noise, while that between $\mathcal{A}_{m}$ and ICV is caused by high-dimensionality. Note that we only observe the ESD of PAV, whereas the ESDs of both ICV and $\mathcal{A}_{m}$ are underlying. Our goal is to estimate the ESD of ICV. As we explained below Theorem 2.1, such 

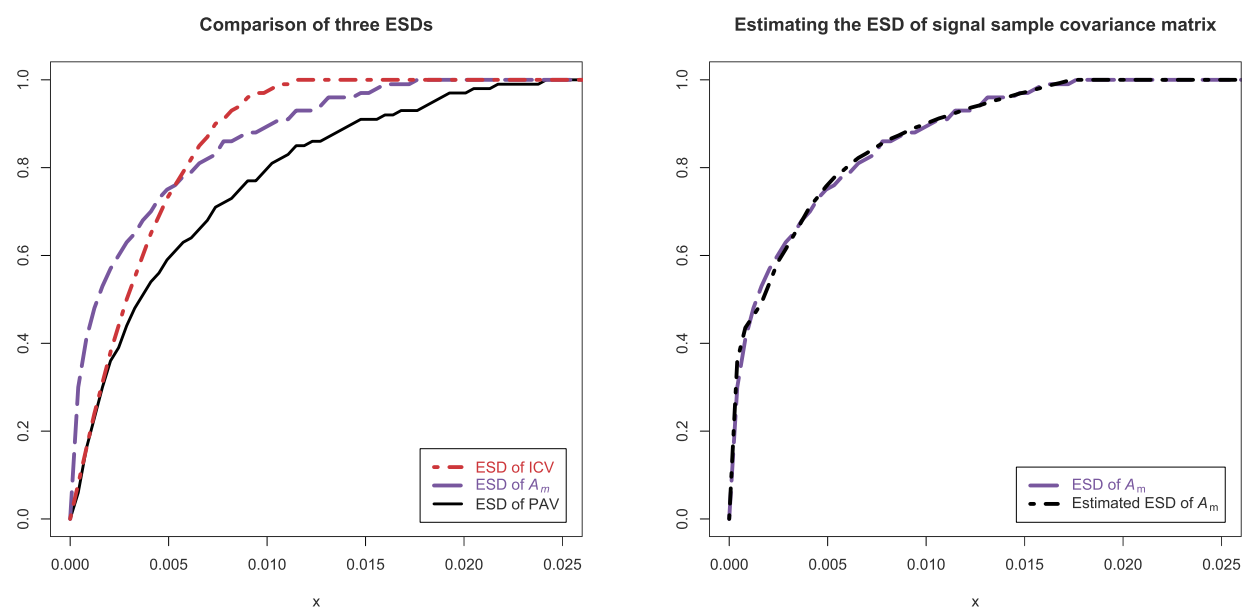

FIG. 1. Approach I-Step 1: Estimation of the empirical spectral distribution of the signal sample covariance matrix $\mathcal{A}_{m}$ based on synchronous noisy observations under model (3.1). The dimension $p=100$, and the observation frequency $n=23,400$.

a goal does not require estimating the ESD of $\mathcal{A}_{m}$. Here, we still estimate this ESD to illustrate the application of Corollary 2.1. The estimation of the ESD of $\mathcal{A}_{m}$ is conducted in the first step, and the result is shown in the right plot of Figure 1. The second step estimates the ESD of ICV, with the result given in Figure 2.

Figures 1 and 2 show that the ESDs of both $\mathcal{A}_{m}$ and ICV can be estimated quite well.

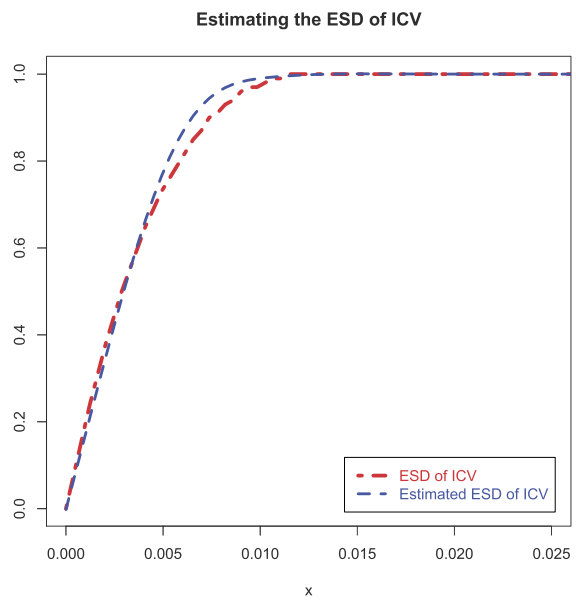

FIG. 2. Approach I-Step 2: Estimation of the empirical spectral distribution of targeting ICV matrix. 

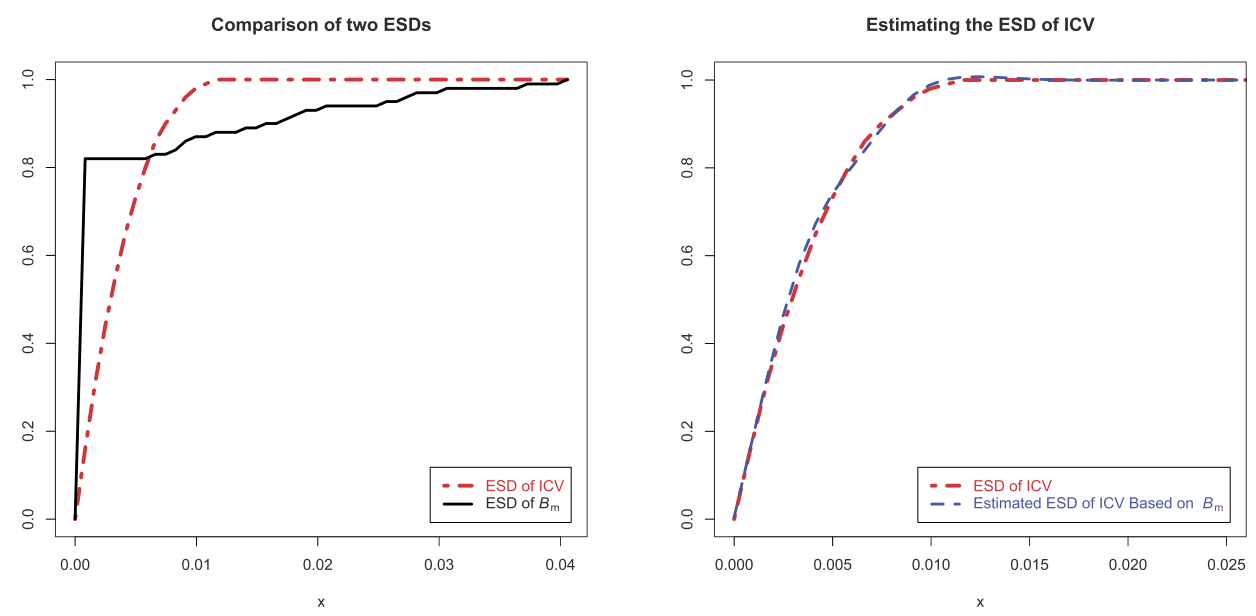

FIG. 3. Approach II: Estimation of the empirical spectral distribution of the targeting ICV matrix based on synchronous noisy observations under model (3.1). The dimension $p=100$ and the observation frequency $n=23,400$.

3.1.2. Estimation using Approach II: Synchronous setting. We now apply Approach II to estimate the ESD of ICV. According to Theorem 2.3, asymptotically, the ESD of $\mathcal{B}_{m}$ is related to that of ICV through the standard Marčenko-Pastur equation. This allows us to directly apply existing algorithms that are developed to invert the Marčenko-Pastur equation to estimate the ESD of ICV, and in the below we adopt the algorithm proposed in El Karoui (2008).

Specifically, set the window length $k$ in defining $\mathcal{B}_{m}$ to be $\left\lfloor 1.5 n^{0.6}\right\rfloor=627$. Discretize the ESD of ICV as (3.4). According to Theorem 2.3, the Stieltjes transform of the ESD of $\mathcal{B}_{m}$, denoted by $m_{\mathcal{B}_{m}}(z)$, should approximately satisfy equation (2.20) with $H$ replaced with the ESD of ICV. In other words, we again expect the approximation errors

$$
e_{j}^{\prime \prime \prime}:=m_{\mathcal{B}_{m}}\left(z_{j}\right)-\sum_{k=1}^{K} \frac{c_{k}}{x_{k}\left(1-y\left(1+z_{j} m_{\mathcal{B}_{m}}\left(z_{j}\right)\right)\right)-z_{j}}
$$

to be small. Thus, again, we estimate the weights $c_{k}$ 's by minimizing the approximation errors $e_{j}^{\prime \prime \prime}$ as in (3.3).

The estimation results are given in Figure 3. Again, we see from the left plot that the ESD of $\mathcal{B}_{m}$ clearly differs from the (latent unobserved) ESD of ICV, yet the right plot shows that we can estimate this latent distribution well.

3.2. When observations are asynchronous. We now consider a setting where the observations are asynchronous. More specifically, for each stock $j=1, \ldots, p$, we simulate a Poisson process of rate 23,400 denoted by $\left\{\eta_{i}^{j}\right\}_{i=0,1, \ldots}$. Because the Poisson processes $\left\{\eta_{i}^{j}\right\}_{i=0,1, \ldots}$ are to be generated independently, almost surely, 
$\eta_{i_{1}}^{j_{1}} \neq \eta_{i_{1}}^{j_{2}}$ for all $1 \leq j_{1} \neq j_{2} \leq p$ and $0<i_{1}, i_{2}$, namely, the observation times are all different for different stocks. Figure 8 in the supplementary article Xia and Zheng (2018) shows the observation times for three stocks generated in such a way during the first ten seconds. The observation times are highly irregularly spaced: there can be several seconds without a single observation, while there can also be several observations within a single second. Furthermore, because observation times for different stocks are generated independently, different stocks are observed in a rather unsynchronized manner, making the estimation of covariances difficult. For this reason, a synchronization procedure needs to be carried out before we apply either Approach I or II.

Before we discuss how to synchronize data, we first continue with the simulation design. To generate the latent process $\left(X_{t}\right)$, because of the asynchronicity and high-dimensionality (we are dealing with $p=100$ independent Poisson processes and, consequently, roughly $23,400 \times 100$ distinct observation times), there is a real technical difficulty in incorporating interactions among component processes in the data generating process. We adopt the following simplified setting to facilitate the simulation. Observe that the results in the previous subsection are achieved when the component processes have dependence, so we believe our methods would still work when there is asynchronicity and dependence. The simplified setting is as follows:

$$
d \mathbf{X}_{t}=\phi_{t} \mathbf{D}^{1 / 2} d \mathbf{W}_{t},
$$

where both $\left(\phi_{t}\right)$ and $\mathbf{D}$ are as in the previous subsection.

Our observations are

$$
Y_{\eta_{i}^{j}}^{j}=X_{\eta_{i}^{j}}^{j}+\varepsilon_{i}^{j},
$$

where $\varepsilon_{i}^{j}$ are i.i.d. $N(0,0.0002)$.

Now we discuss how to synchronize data. We adopt the previous tick method explained in Section 2.4. More specifically, we choose an equally spaced grid $\left\{t_{i}\right\} \subseteq[0,1]$, and for each $t_{i}$, for each $j=1, \ldots, p$, let

$$
\tau_{i}^{j}=\max \left\{\eta_{k}^{j}: \eta_{k}^{j} \leq t_{i}\right\} .
$$

We then proceed as if we observe $Y_{\tau_{i}^{j}}^{j}$ at time $t_{i}$. As we explained in Section 2.4, because $\tau_{i}^{j} \neq t_{i}$, such a synchronization procedure introduces an additional error $X_{\tau_{i}^{j}}^{j}-X_{t_{i}}^{j}$.

3.2.1. Estimation using Approach I: Asynchronous setting. The additional error $X_{\tau_{i}^{j}}^{j}-X_{t_{i}}^{j}$ that the synchronization procedure induces depends on the latent process. For this reason, our independence assumption between the noise and the latent process (B.ii) is violated. To alleviate this problem, we synchronize less fre- 
Comparison of three ESDs

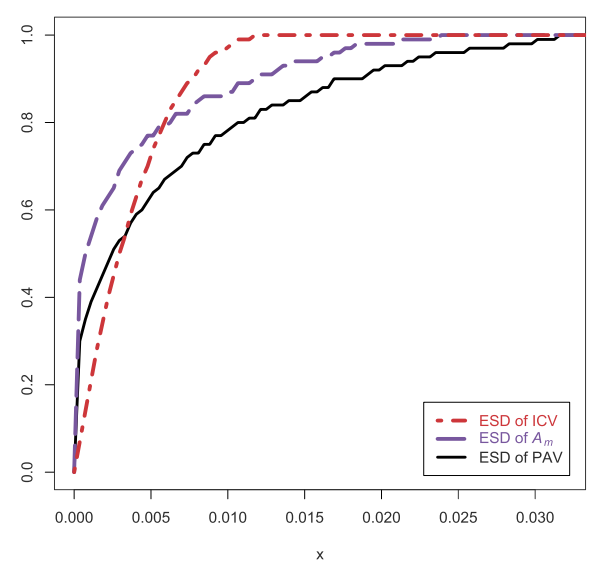

Estimating the ESD of signal sample covariance matrix

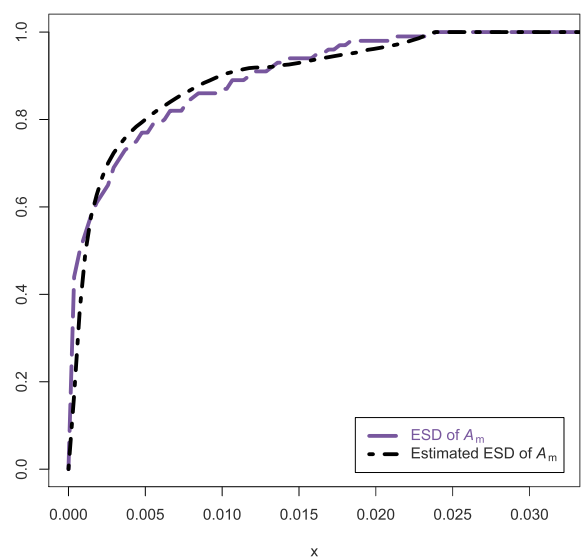

FIG. 4. Approach I-Step 1: Estimation of the empirical spectral distribution of the signal sample covariance matrix $\mathcal{A}_{m}$ based on asynchronous noisy observations under model (3.5). The dimension is $p=100$. The synchronization frequency is 4 seconds, which leads to $n=23,400 / 4=5850$ observations.

quently so that the signals $X_{\tau_{i}^{j}}^{j}-X_{\tau_{i-1}^{j}}^{j}$ tend to be bigger and better approximate the true signals $X_{t_{i}}^{j}-X_{t_{i-1}}^{j}$. More specifically, we choose the equally spaced grid to be $\left\{t_{i}=4 i / 23,400\right\}$-in other words, we synchronize once every four seconds. Then, following the estimation procedure in Section 3.1.1, we have the following results, see Figures 4 and 5.

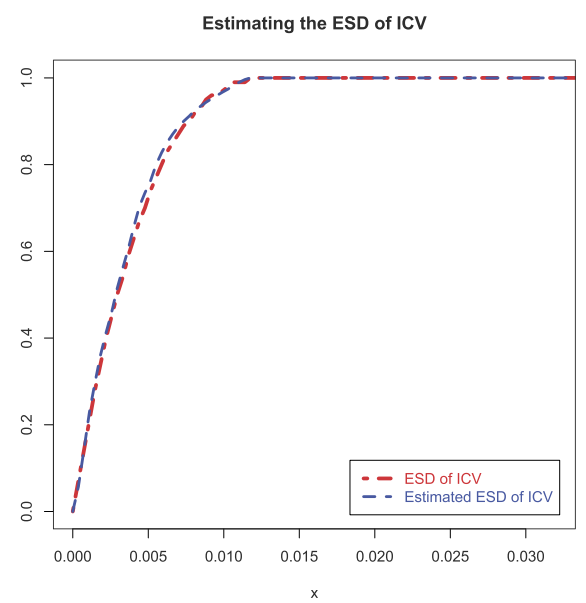

FIG. 5. Approach I-Step 2: Estimation of the empirical spectral distribution of the targeting ICV matrix. 

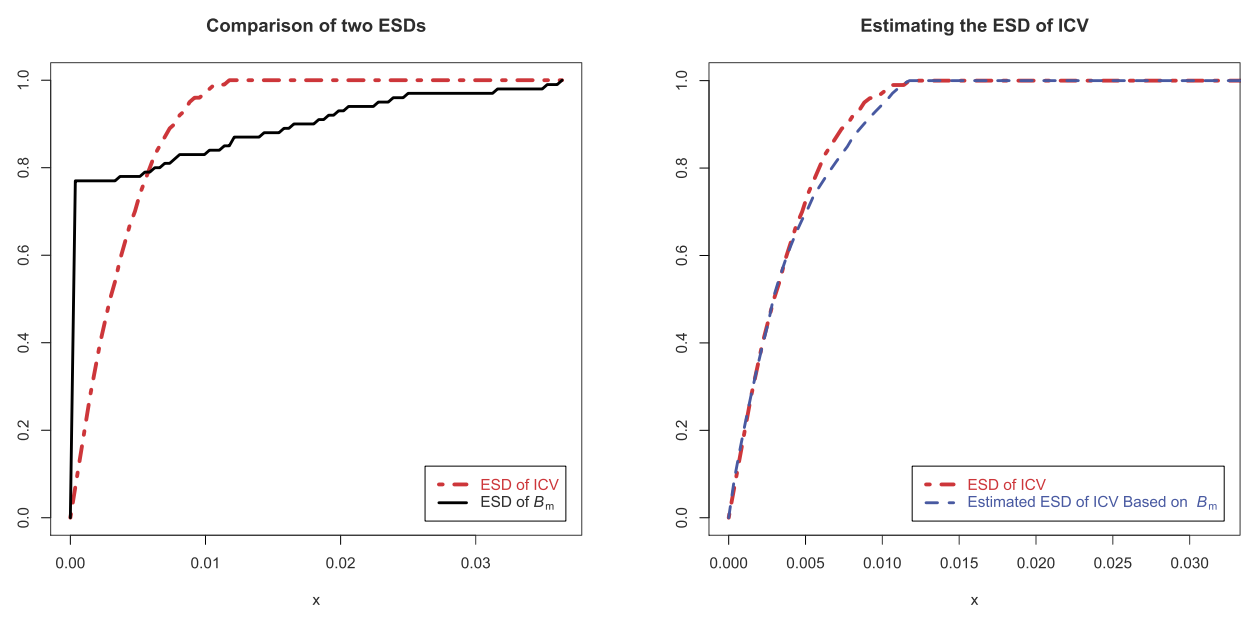

FIG. 6. Approach II: Estimation of the empirical spectral distribution of the targeting ICV matrix based on asynchronous noisy observations under model (3.5). The dimension is $p=100$. The synchronization frequency is one second, which leads to $n=23,400$ observations.

We see that in such a highly asynchronous noisy observation setting, Approach I still works quite well.

3.2.2. Estimation using Approach II: Asynchronous setting. Approach II relies on Theorem 2.3, which allows for dependence in the noise process and between the noise and price process. For this reason, it is more robust than Approach I, and we can synchronize more frequently. In the estimation below, we choose to synchronize once every second; that is, the time grid is taken to be $\left\{t_{i}=i / 23,400\right\}$. Then, following the estimation procedure in Section 3.1.2, we obtain the following results, see Figure 6.

Again, we see that in such an asynchronous noisy observation setting, Approach II works quite well.

3.3. Discussions about the two approaches. The two approaches have their own pros and cons.

- Approach I is more widely applicable to noisy observation situations. Moreover, in our particular application, because the window width in defining the PAV matrix is $O(\sqrt{n})$, which is of lower order than that for $\mathcal{B}_{m}$ in Approach II, Approach I essentially has a larger "effective sample size." This approach, however, is more sensitive to the model assumptions. In particular, in the asynchronous observation setting, because of the additional error introduced by asynchronicity, we may need to synchronize less frequently.

- Approach II is more direct because it only involves a one-step estimation procedure. It is also more robust because it allows for rather general dependence 
structures in the noise process, both cross-sectional and temporal, and even dependence between the noise and price process. For this reason, in the asynchronous setting in Section 3.2, we can use a higher synchronization frequency than for Approach I. A major drawback of Approach II is that it relies heavily on some special properties of the particular setting under study and hence may not be applicable to other noisy observation situations.

Finally, while in the estimation above we largely adapt the algorithms proposed by El Karoui (2008) to fit our setting, other algorithms such as those in Mestre (2008), Bai, Chen and Yao (2010) and Ledoit and Wolf (2015) can also be adapted.

4. Conclusion. Motivated by the inference about the spectral distribution of the ICV matrix based on high-frequency noisy data:

- we establish an asymptotic relationship that describes how the spectral distribution of the signal sample covariance matrices depends on that of the sample covariance matrices constructed from noisy observations;

- using further a (generalized) connection between the spectral distribution of the signal sample covariance matrices and that of the population covariance matrix, we propose a two-step procedure that can consistently estimate the spectral distribution of ICV for a class of diffusion processes;

- we further develop an alternative approach that possesses several desirable properties: it is more robust, it eliminates the effects of microstructure noise, and the asymptotic relationship that enables the consistent estimation of the spectral distribution of ICV is the standard Marčenko-Pastur equation.

- numerical studies demonstrate that our proposed methods work well, under both synchronous and asynchronous observation settings.

Acknowledgments. We are very grateful to the Editor, the Associate Editor and the anonymous referees for their valuable comments and constructive suggestions, which led to a substantial improvement of this paper.

\section{SUPPLEMENTARY MATERIAL}

Supplement to "On the inference about the spectral distribution of highdimensional covariance matrix based on high-frequency noisy observations" (DOI: 10.1214/17-AOS1558SUPP; .pdf). Due to space constraints, the proofs of Theorems 2.1, 2.2 and 2.3 are given in the supplementary article Xia and Zheng (2018).

\section{REFERENCES}

AÏT-SAHAliA, Y., FAn, J. and LI, Y. (2010). The leverage effect puzzle: Disentangling sources of bias at high frequency. J. Financ. Econ. 109 224-249.

AÏT-SAHAliA, Y., FAN, J. and XIU, D. (2010). High-frequency covariance estimates with noisy and asynchronous financial data. J. Amer. Statist. Assoc. 105 1504-1517. MR2796567 
Andersen, T. G. and Bollerslev, T. (1998). Answering the skeptics: Yes, standard volatility models do provide accurate forecasts. Internat. Econom. Rev. 39 885-905.

Andersen, T. G., Bollerslev, T., Diebold, F. X. and Labys, P. (2001). The distribution of realized exchange rate volatility. J. Amer. Statist. Assoc. 96 42-55. MR1952727

BAI, Z., CHEN, J. and YAO, J. (2010). On estimation of the population spectral distribution from a high-dimensional sample covariance matrix. Aust. N. Z. J. Stat. 52 423-437. MR2791528

BAI, Z. and SilversteIN, J. W. (2010). Spectral Analysis of Large Dimensional Random Matrices, 2nd ed. Springer, New York. MR2567175

BARNDORFF-NIELSEN, O. E. and SHEPHARD, N. (2002). Econometric analysis of realized volatility and its use in estimating stochastic volatility models. J. R. Stat. Soc. Ser. B. Stat. Methodol. 64 253-280. MR1904704

Barndorff-Nielsen, O. E., Hansen, P. R., Lunde, A. and Shephard, N. (2008). Designing realized kernels to measure the ex post variation of equity prices in the presence of noise. Econometrica 76 1481-1536. MR2468558

Barndorff-Nielsen, O. E., Hansen, P. R., Lunde, A. and Shephard, N. (2011). Multivariate realised kernels: Consistent positive semi-definite estimators of the covariation of equity prices with noise and non-synchronous trading. J. Econometrics 162 149-169. MR2795610

Christensen, K., Kinnebrock, S. and PodolskiJ, M. (2010). Pre-averaging estimators of the ex-post covariance matrix in noisy diffusion models with non-synchronous data. J. Econometrics 159 116-133. MR2720847

Dozier, R. B. and Silverstein, J. W. (2007a). On the empirical distribution of eigenvalues of large dimensional information-plus-noise-type matrices. J. Multivariate Anal. 98 678-694. MR2322123

El KAROUI, N. (2008). Spectrum estimation for large dimensional covariance matrices using random matrix theory. Ann. Statist. 36 2757-2790. MR2485012

EL KAROUI, N. (2009). Concentration of measure and spectra of random matrices: Applications to correlation matrices, elliptical distributions and beyond. Ann. Appl. Probab. 19 2362-2405. MR2588248

EL KAROUI, N. (2010a). High-dimensionality effects in the Markowitz problem and other quadratic programs with linear constraints: Risk underestimation. Ann. Statist. 38 3487-3566. MR2766860

El KAROUI, N. (2010b). On information plus noise kernel random matrices. Ann. Statist. 38 31913216. MR2722468

EL KAROUI, N. (2013). On the realized risk of high-dimensional Markowitz portfolios. SIAM J. Financial Math. 4 737-783. MR3118251

Gloter, A. and JACOD, J. (2001). Diffusions with measurement errors. II. Optimal estimators. ESAIM Probab. Stat. 5 243-260. MR1875673

Hachem, W., Loubaton, P., Mestre, X., Najim, J. and Vallet, P. (2012). Large information plus noise random matrix models and consistent subspace estimation in large sensor networks. Random Matrices Theory Appl. 1 1150006, 51. MR2934713

HANSEN, P. R. and LUNDE, A. (2006). Realized variance and market microstructure noise. J. Bus. Econom. Statist. 24 127-218. MR2234447

JACOD, J., LI, Y. and ZHENG, X. (2017). Statistical properties of microstructure noise. Econometrica. To appear. Available at SSRN, http://ssrn.com/abstract=2212119.

JACOD, J. and PROTTER, P. (1998). Asymptotic error distributions for the Euler method for stochastic differential equations. Ann. Probab. 26 267-307. MR1617049

Jacod, J., Li, Y., Mykland, P. A., PodolskiJ, M. and Vetter, M. (2009). Microstructure noise in the continuous case: The pre-averaging approach. Stochastic Process. Appl. 1192249 2276. MR2531091

LEDOIT, O. and WoLF, M. (2015). Spectrum estimation: A unified framework for covariance matrix estimation and PCA in large dimensions. J. Multivariate Anal. 139 360-384. MR3349498 
LiU, L. Y., Patton, A. J. and ShePPARD, K. (2015). Does anything beat 5-minute RV? A comparison of realized measures across multiple asset classes. J. Econometrics 187 293-311. MR3347308

MARČENKO, V. A. and PASTUR, L. A. (1967). Distribution of eigenvalues in certain sets of random matrices. Mat. Sb. 72 507-536. MR0208649

MCNeil, A. J., Frey, R. and Embrechts, P. (2005). Quantitative Risk Management: Concepts, Techniques and Tools. Princeton Univ. Press, Princeton, NJ. MR2175089

Mestre, X. (2008). Improved estimation of eigenvalues and eigenvectors of covariance matrices using their sample estimates. IEEE Trans. Inform. Theory $\mathbf{5 4}$ 5113-5129. MR2589886

MyKLAND, P. A. and ZHANG, L. (2006). ANOVA for diffusions and Itô processes. Ann. Statist. 34 1931-1963. MR2283722

PodolskiJ, M. and VetTER, M. (2009). Estimation of volatility functionals in the simultaneous presence of microstructure noise and jumps. Bernoulli 15 634-658. MR2555193

UBUKATA, M. and OYA, K. (2009). Estimation and testing for dependence in market microstructure noise. J. Financ. Econom. 7 106-151.

WANG, C. D. and MYKLAND, P. A. (2014). The estimation of leverage effect with high-frequency data. J. Amer. Statist. Assoc. 109 197-215. MR3180557

XIA, N. and ZhENG, X. (2018). Supplement to "On the inference about the spectral distribution of high-dimensional covariance matrix based on high-frequency noisy observations." DOI:10.1214/17-AOS1558SUPP.

XIU, D. (2010). Quasi-maximum likelihood estimation of volatility with high frequency data. $J$. Econometrics 159 235-250. MR2720855

ZHANG, L. (2006). Efficient estimation of stochastic volatility using noisy observations: A multiscale approach. Bernoulli 12 1019-1043. MR2274854

ZHANG, L. (2011). Estimating covariation: Epps effect, microstructure noise. J. Econometrics 160 33-47. MR2745865

Zhang, L., Mykland, P. A. and AïT-Sahalia, Y. (2005). A tale of two time scales: Determining integrated volatility with noisy high-frequency data. J. Amer. Statist. Assoc. 100 1394-1411. MR2236450

ZHENG, X. and LI, Y. (2011). On the estimation of integrated covariance matrices of high dimensional diffusion processes. Ann. Statist. 39 3121-3151. MR3012403

SCHOOL OF STATISTiCS AND MANAGEMENT, SHANGHAI

KEY LABORATORY OF FINANCIAL INFORMATION TECHNOLOGY

SHANGHAI UNIVERSITY OF FINANCE AND ECONOMICS

DEPARTMENT OF INFORMATION SYSTEMS BUSINESS STATISTICS AND OPERATIONS MANAGEMENT

777 Guo Ding RoAd, 200433

Hong Kong University of SCIENCE AND TECHNOLOGY

Clear Water Bay, Kowloon

HONG KONG

CHINA

E-MAIL: xhzheng@ust.hk

E-MAIL: xia.ningning@mail.shufe.edu.cn 